\title{
Simulation and Optimization of Medicine Service System Based on Random Service System
}

\author{
Yu-heng Song, Yao-yao Wei* \\ School of Management, University of South China, Hengyang, China. \\ Email: 24359048@qq.com
}

\begin{abstract}
As one of the direct contact windows in medical processes, the medicine services have attracted lots of attentions. Small pharmacy scale and unreasonable setting of the service process cause patients to spend long waiting time for taking medicines. Taking the Second Affiliated Hospital of the University Of South China as the study object, firstly, through on-site observation and investigation of the current medicine service process of the hospital, the patient demands are measured and evaluated, and the layouts of pharmacy facilities and service mechanism are analyzed. Secondly, by describing the structure and the operation characteristics of the outpatient system, we construct a simulation model of the medicine service system based on Random Service System (RSS). Finally, the bottlenecks that limit the efficiency of the system are shown by the simulation results of the models and then system can be optimized effectively.
\end{abstract}

Keywords: Optimization of medicine service system, random service system.

\section{Introduction}

Under the background of the health care reform in China, the medicine service is developing rapidly and facing a number of chances and challenges. With the increasing demand for the medicine service, patients give a higher request to the level and content of medicine service system than ever. As one of the direct contact windows in medical processes, the medicine services have attracted lots of attentions. Small pharmacy scale and unreasonable setting of the service process cause patients to spend long waiting time for taking medicines. How to correctly describe the layout and operation mode of medicine service system, and to find bottlenecks that limit the efficiency of service system is a major issue for the pharmacy management department.

\subsection{The Construction of FlexSim Based on Random Service System}

In recent years, with the acceleration of living rhythm and increasing competition of medicine service industry, the masses gives a higher request to the quality of medical care, hospital environment, and clinic time. As an important window of medicine service, pharmacy management level is closely related to patients' interest and has an important effect on the quality of hospital service and overall image. The phenomenon of "three long and one short"(the long time of registration, waiting doctor and payment, the short time for treating) still exist in hospital today, while at the same time long waiting time for taking medicine to the pharmacy poses another major problem which has direct impact on the hospital management level and the satisfaction of patients. Therefore, it's of great practical significance to explore the optimization problem of medicine service system with the scientific theory and normative method.

WANG jianyun and WU shaokun pointed out that drug management regulations and process, staff management and information construction are the main aspects of error accidents at pharmacy [1]. Based on the digital model of pharmacy, Pedersen $\mathrm{C}$ analyzes the current situation and existing problems of pharmacy and puts forward the theory that constructs the digital pharmacy model and combines information technology and automation technology with pharmacy practice activities [2]. CHEN ke proposed an optimization plan for the disadvantages of traditional procedure in medicine service based on information platform, as well as develop and establish the self -service system based on the ideas of "parallelization and integration"[3]. LI jun and KANG xiangwei studied the advantages of applying automatic management system in pharmacies [4]. K. Lynee James, et al explored the automatic 
management system and its application effects [5]. J Xie, QM He, X Zhao investigated the stability of a priority queuing system with customer transfer [6]. In 2008, Dieter Fiems and Tom Marrtes studied queuing system with different types of service termination [7].

\section{Methodology}

In conclusion, medicine service system with complex program faces a variety of patients, so it is difficult to model in the traditional way. The way of traditional modeling can't dynamically reflect the real system and its complex environment. In addition, it can't continuously and effectively analyze and optimize the real system in real time, and get a more feasible solution. Decision technology based on Random Service System can provide a better theory for the optimization of the medicine service system than ever.

Random Service System (RSS) is a system that the statistical rules of quantity index are obtained through the statistical research of service object's arrival and service time. According to these rules, the structure of the service system will be improved or the service object will be reorganized to meet the needs of the service objects. Most of RSS belongs to Direct Event System (DES). An internal state can change into a variety of states. Zhu, et al investigated the configuration of distribution center and facility layout by using FlexSim basing on RSS, and found bottlenecks that limit the efficiency of system service by analyzing the simulation output. Medicine service system is a typical random service system. Medical disputes and the demand for medicine service are randomly occurring (Patients arrive at the pharmacy randomly and wait for a long time to get the medicine), the service capability and its system state of medicine system are influenced by the change of demands, which have the characteristics of strong randomness.

Hence, based on the RSS modeling theory and FlexSim simulation optimization technique, the paper presents a method to optimize the medicine service system. Taking the Second Affiliated Hospital of the University Of South China as the study object, we observe the random features and establish the model of the medicine service system, run simulation with real data, and continuously and dynamically analyze and optimize the system according to the quantized data by simulation.

\section{$3 \quad$ Results}

The simulation of medicine service system and optimization principle is based on Random Service System.

\subsection{Model}

Random service system

Medicine Service System as shown, the paper takes The Second Hospital University Of South China as the study object, and mainly focuses on medicine service system which really restores the medicine service process based on RSS and FlexSim software. When the patients arrive at the hospital randomly, the first step is to get the prescription after seeing a doctor. When they reach the medicine service area with the prescription, if pharmacy is occupied, they will automatically queue up at the end of the queue to wait for being serviced. The patients continue to wait for long time after the prescription is delivered to the pharmacy. The arrival time that patient gets the medicine is random due to the difference in clinic time. Pharmacists begin to make up a prescription with the prescription. Because of the differences of prescription content, its service hours are also random. The patients take the medicine and leave after the dispensing is completed. 


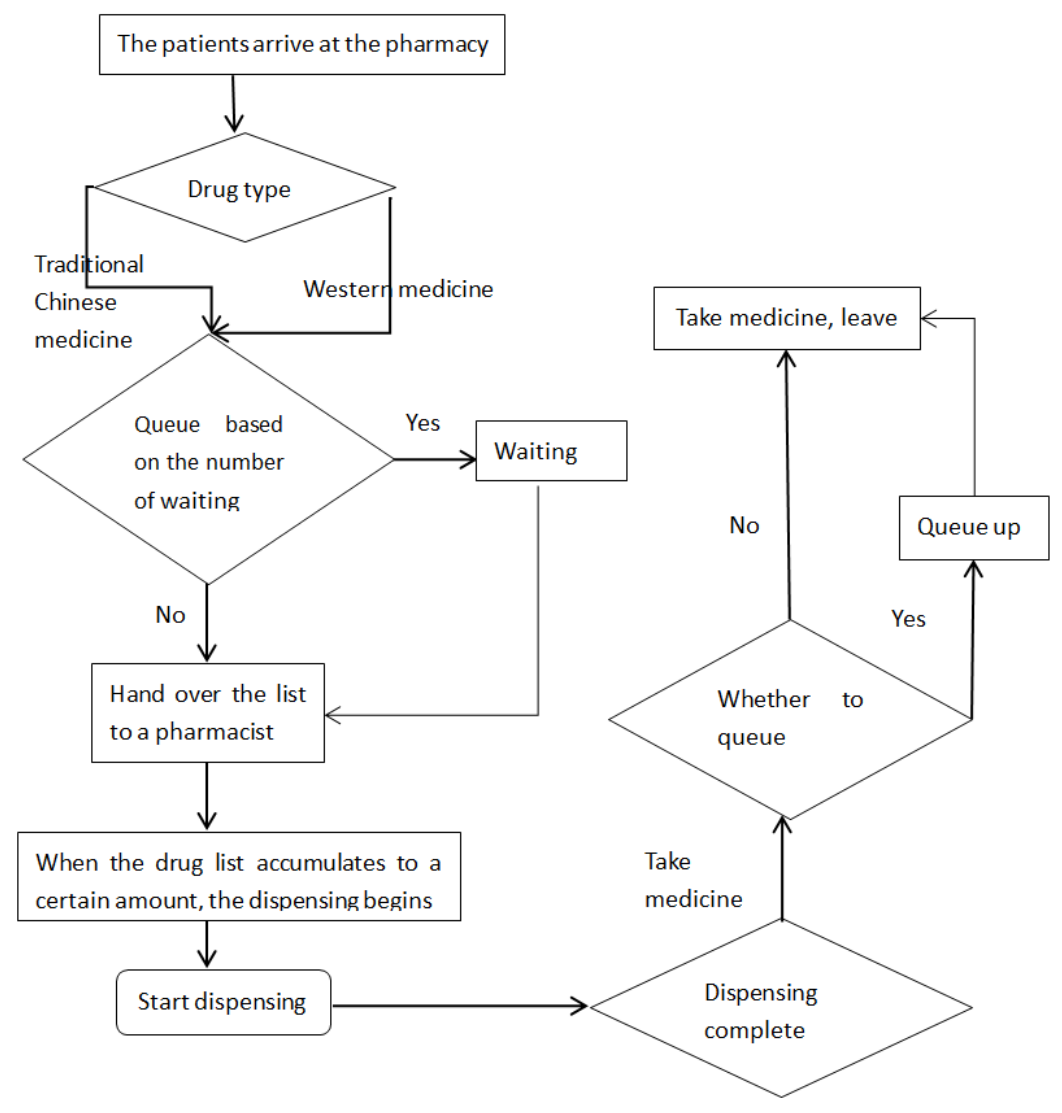

Figure 1. The abstract model of medicine service system

\subsection{Simulation and Optimization}

(1)Through the actual investigation and data collection, the paper understands the overall hospital service efficiency of the status, and fits the statistic rule of patient arrival and medicine service based on ExpetFit software.

(2)Based on the analysis of the characteristics of the medicine service system, this paper analyzes and describes the service flow of the pharmacy, and constructs the abstract structure and service model of the system based on the RSS theory.

(3) The model based on RSS is transformed into simulation model by FlexSim simulation technology. According to the current medicine service mode, the service mode chart is drawn, and the reorganization target is determined. It includes adding automatic dispensing device, sharing information between dispensaries and clinics, adding an electronic prescription, reducing the error rate, improving the service quality and level of outpatient pharmacy, which sets different requirements for different phases and quantifies it. The real data is introduced to analyze the operation condition under the steady states, and the bottlenecks of solving the problem of queuing congestion and high error rate is found. During the "discovery-improvement", an effective system optimization scheme is proposed.

\section{Example}

Taking the Second Affiliated Hospital of the University Of South China for example, pharmacy adopts a traditional manual dispensing mode when the patient's prescription information is collected from the window. The pharmacists in the pharmacy manually begin to place the drug according to the prescription information, and send the medicines to the drug dispensing personnel in the window. Next, the pharmacists in window check prescription and fill in the dosage. Finally, the medicine is given to the patient and medication notes are given to the patient. There are mostly outdated equipped and inefficient 
medicines picking system in traditional pharmacies, which make the patient wait for a long time to get the medicine and increase the physician-patient disputes. Based on actual research data, the patients 'arrival rate is 5 minutes per person; the time from the doctor writes a prescription to the patient's arrival at the pharmacy is 20 minutes; outpatient pharmacy dispensing time is 8 minutes; the average time of delivering the drug to the patient is 10 minutes.

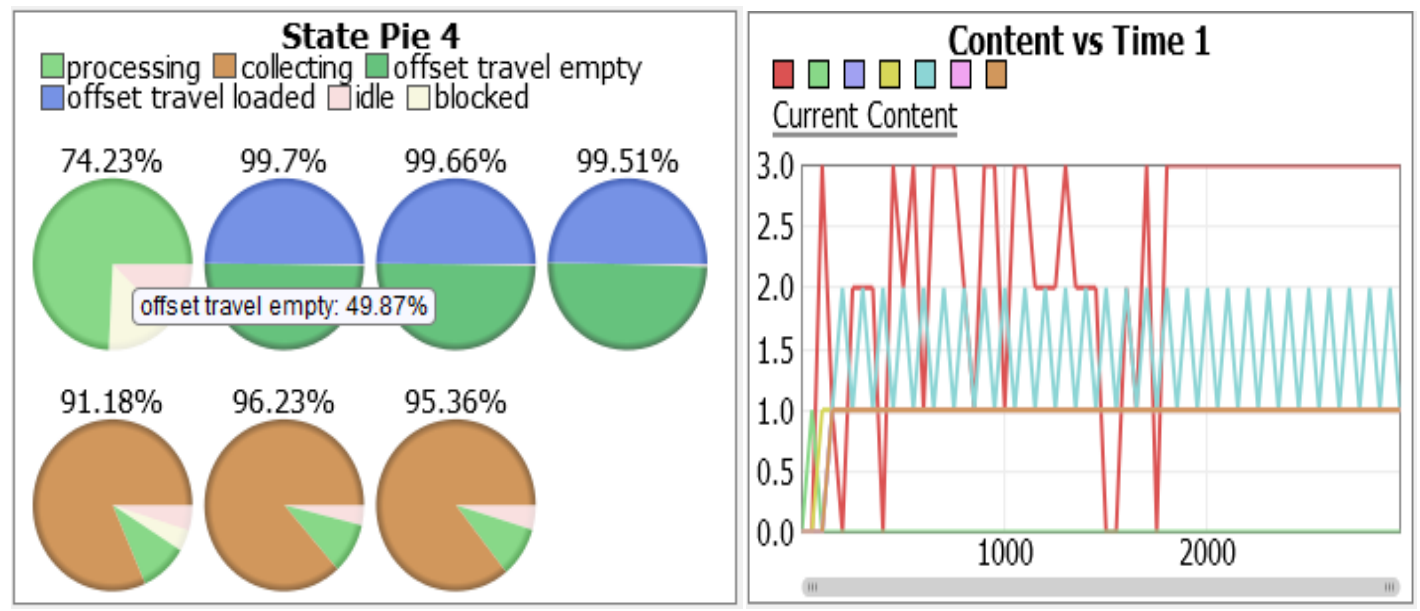

Figure 2. The screenshot of simulation data before MSS optimizing

With the increase of the number of patients and pharmacists' workload, the dispensing efficiency of pharmacists is decreased with the increase of the number of patients. Pie 2, Pie 3 and Pie 4 are up to $90 \%$ (pharmacists reach the limit of maximum workload and the error rate rises linearly.) Pie 5, Pie 6 and Pie 7 are more than $90 \%$ (the workload of the drug station exceeds the acceptable capacity, and the efficiency begins to decrease. These experimental data shows that traditional drug delivery model needs manual inspection and identification pharmacists have heavy workload and high error rate, phenomenon of queuing is serious, which affect the overall service efficiency of the hospital.

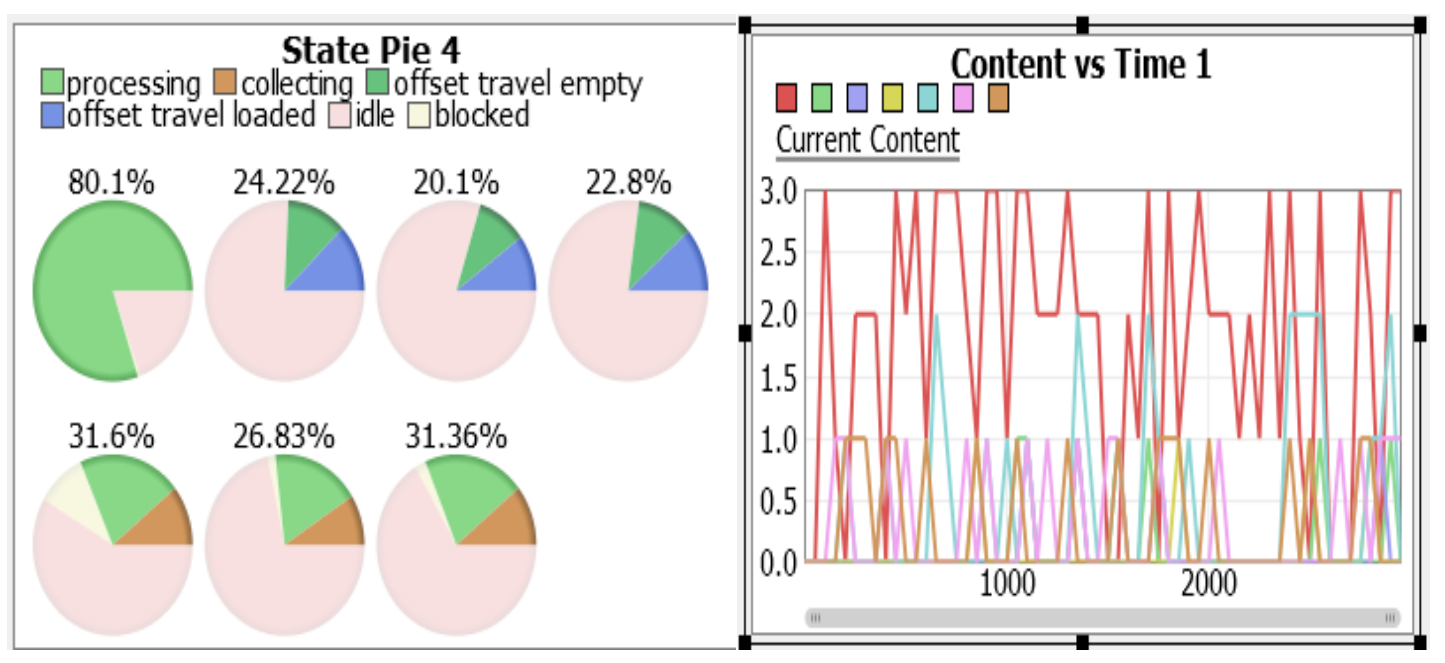

Figure 3. The screenshot of simulation data after MSS optimizing

By improving the dispensing system, medicine taking and dispensing mode in the original medical service system, we optimize the medicine service system, reduce the workload of pharmacists, reduce drug dispensing errors, and optimize drug management, while at the same time it has succeeded in shortening the waiting time for patient to take the medicine, improving the hospital outpatient pharmacy service and management level. 


\subsection{Initial Data Include:}

(1) The time interval of the patient's arrival obey index distribution, Average 10 (seconds).

(2) The arrival laws of different kinds of patients obey uniform distribution and index distribution.

(3) The patient's arrival law obeys discrete uniform distribution.

(4) The dispensing service time obeys normal distribution, average 8 (seconds) Standard deviation is 3 (seconds).

(5) System service time obeys normal distribution, averaging 10 (seconds) and standard deviation 3 (seconds).

(6) The maximum capacity of each waiting queue is 200.

(7) The total time of simulation is greater than equal to 50000 seconds.

Table 1. Major entities and related annotations

\begin{tabular}{l|l|l}
\hline The entity name & System elements & Relevant comments \\
\hline Source1,2 & The hospital hall & The stochastic patients' arrival \\
\hline Queue & Queue area & Random queue rule \\
\hline Source3 & Medicine storage area & Dispensing process \\
\hline Rack1,2,3 & Drug classification area & The sorting and matching of drugs \\
\hline Robot1,2,3 & The pharmacist & Drug sorting and handling \\
\hline Combiner $1,2,3$ & Outpatient pharmacy window & $\begin{array}{l}\text { The patient takes the drug to the } \\
\text { pharmacy door to match the } \\
\text { medicine }\end{array}$ \\
\hline
\end{tabular}

\section{Discussion}

This paper simulates and analyzes the model of medicine service system based on RSS, and finds out the random arrival through the analysis and research of the actual survey data. It imports data based on RSS Andre establish FlexSim simulation model, and finds out bottlenecks that limit the efficiency of medicine service system which helps improve the efficiency of the medicine system service and optimization of drug management, and provides a reference for the comprehensive development of the hospital.

Acknowledgements. The paper is supported by Hunan Education Department Excellent Youth Project (No. 17B236);Research Learning and innovative Experimental Plan of University of South China Department Project (2017XJYZ064); the research is also supported by the Key Discipline of Management Science and Engineering of USC.

\section{References}

1. Wangjianhui, Wushaokun. (2010). Errors Accidents and Management of Hospital Pharmacy[J]. China Pharmaceutical, 19(16), 44-45 (In Chinese). http://www.cqvip.com/Main/Detail.aspx?id=35057808

2. Pedersen, C. A., \& Gumpper, K. F.(2015). Ashp national survey on informatics: assessment of the adoption and use of pharmacy informatics in u.s. hospitals - 2007. American journal of health-system pharmacy: AJHP: official journal of the American Society of Health-System Pharmacists, 72(8), 636-55. https://academic.oup.com/cid

3. CHEN Ke, GUAN Li-hua \& WANG Jun. (2012). Optimization Research of Outpatient Service Process Based on the Self-help Service [J]. China Digital Medicine, 07(10), 21-23 (In Chinese). http://kns.cnki.net/kcms/ detail/detail.aspx?dbcode=CJFD\&filename=YISZ201210011\&dbname=CJFDLAST2015

4. LIANG jun, KANG xiang-wei. (2017) Analysis of automatic pharmacy management system in outpatient pharmacy [J]. Cardiovascular Disease Journal of Integrated Traditional Chinese and Western Medicine (Electronic), 22(5):450-451. (In Chinese) http://d.wanfangdata.com.cn/Periodical/zxyjhxxgbdzzz201722004 
5. James, K. L., Barlow, D., Bithell, A., Hiom, S., Lord, S., \& Pollard, M., et al. (2013). The impact of automation on workload and dispensing errors in a hospital pharmacy. International Journal of Pharmacy Practice, 21(2), 92-104. http://onlinelibrary.wiley.com/doi/10.1111/j.2042-7174.2012.00238.x/abstract

6. Xie, J., He, Q. M., \& Zhao, X. (2008). Stability of a priority queuing system with customer transfers. Elsevier Science Publishers B. V. http://www.sciencedirect.com/science/article/pii/S0167637708000849

7. Fiems, D., Maertens, T., \& Bruneel, H. (2008). Queuing systems with different types of server interruptions. European Journal of Operational Research, 188(3), 838-845. http://www.sciencedirect.com/science/article/pii/ S0377221707004894

8. Miller, D. A., Zarowitz, B. J., Petitta, A., \& Wright, D. B. (1993). Pharmacy technicians and computer technology to support clinical pharmacy services. Am J Hosp Pharm, 50(5), 929-934. http://europepmc.org/abstract/ $\mathrm{MED} / 8506872$

9. HOLDFORD D. A, \& SMITH S. (1997). Improving the quality of outcomes research involving pharmaceutical services. commentary. American Journal of Health-System Pharmacy. http://www.refdoc.fr/ Detailnotice?cpsidt $=2700907$

10.Nanji, K. C., Cina, J., Patel, N., Churchill, W., Gandhi, T. K., \& Poon, E. G. (2009). Overcoming barriers to the implementation of a pharmacy bar code scanning system for medication dispensing: a case study. Journal of the American Medical Informatics Association, 16(5), 645-650. https://academic.oup.com/jamia

11.PEDERSEN CA, GUMPPER KF. (2016) American journal of health-system pharmacy: AJHP[J]. $2244-2264$. http://www.ovid.com/site/catalog/journals/317.jsp

12.National Center for Biotechnology Information, Available: http://www.ncbi.nlm.nih.gov 